\title{
The healthy root pulp, degradation and regeneration
}

\begin{abstract}
Beneath the cell free and cell rich zones of peripheral layers, the dental pulp includes a series of healthy cells, surrounded by a ground substance forming a framework in support of a fibrous and non-fibrous extracellular matrix. Tubular dentin constitutes the outer layers, covering both the crown and $\operatorname{root}(\mathrm{s})$ parts of the teeth. The pulp includes cells that are producing structural fibers (e.g. collagens), and adhesive molecules such as glycosaminoglycans and proteoglycans, fibronectin, membrane lipids (phospholipids and proteolipids) and enzymes (e.g. metalloproteinases and specific inhibitors). Degradation of the root canal pulp is mostly due to proteinases, especially when they are activated by cariogenic bacteria. Vascular and nerve networks promote blood supply, a structure implicated in the tissue repair, releasing bioactive substances producing pain. Stem cells are recruited to heal the wounded pulp tissue. In case of defective stem cell colonies, induced pluripotent stem cells (iPS) allow the regeneration of the dental pulp. Inflammatory and immunocompetent cells contribute to the repair and regeneration of the pulp. Non-carious and carious pulp degeneration contribute to radicular pulp healing under the control of implantated growth factors, together with or without adapted scaffolds.
\end{abstract}

Keywords: dental pulp, stuctural fibers, adhesive molecules, metalloproteinases, induced pluripotent stem cells, pulp regeneration
Volume 10 Issue I - 2019

\author{
Michel Goldberg \\ Department of Oral Biology, Faculty of Fundamental and \\ Biomedical Sciences, Paris Descartes University, France \\ Correspondence: Michel Goldberg, Professor Emeritus, \\ Department of Oral Biology, Paris Descartes University, Faculty \\ of Fundamental and Biomedical Sciences \& INSERM UMR-S \\ I I 24. Stem cells, signalization and prions. 45 rue des saints \\ pères, 75006, Paris, France, Tel 33162676709. \\ Emailmgoldod@gmail.com
}

Received: January 18, 2019 | Published: February 05, 2019
The dental pulp: An introduction to the healthy pulp : the cells and extracellular matrix

\section{Peripheral dentins includes}

1. In the crown, the mantle dentin is formed as an atubular layer $30-150 \mu \mathrm{m}$ thick, poor in sialophosphoproteins (DSPP), and rich in glycosaminoglycans (GAGs).

2. In the root, the Hopewell-Smith and Tomes granular layers constitute the outer limits (each of these layers being $8-15 \mu \mathrm{m}$ thick, with a total thickness approximately of $30 \mu \mathrm{m}$ ). Both layers are located beneath the dentino-enamel or the dentinocementum junctions. In the outer mantle dentin, they contain a few tubules that are bent, and display minute diameter. In the granular layer, the peripheral layers include unfilled interglobular spaces (or calcospheritic structures), apparently empty but actually containing GAGs. These outer layers constitute the limits of the sound circumpulpal dentin (primary and secondary dentins). The pulp volume displays variable thickness, depending the age of the tooth. ${ }^{1}$

The pulp volume vary, depending the age of the patients. For the maxillary incisors and premolars mensurations are approximately $12.4-14.7 \mathrm{~mm}^{3}$, and less for the mandibular incisors and canines $(6.1-$ $\left.14.2 \mathrm{~mm}^{3}\right)$. For the premolars, the mean volume has been evaluated to be between $18.2 \mathrm{~mm}^{3}$ and $14.9 \mathrm{~mm}^{3}$. For the first maxillary molars, the pulp volume was about $68.2 \mathrm{~mm}^{3}$. For the $2^{\text {nd }}$ molar about $44.3 \mathrm{~mm}^{3}$, and for the $3^{\text {rd }}$ molar, measurements indicate values around $22.6 \mathrm{~mm}^{3}$. The mean volume for dental pulp cavities of the mandibular teeth are respectively: $52 \mathrm{~mm}^{3}, 32.9 \mathrm{~mm}^{3}$ and $31 \mathrm{~mm}^{3} .^{2}$ During early tooth formation, the dentin beggins its apposition at $10 \mu \mathrm{m}$ daily rate. Later there is an increse of dentin thickness about 4 micrometers per day, far less in the aged teeth. Odontoblasts and pre-dentin, the zone free and the zone rich layers constitute the outer limits of the dental pulp that is located more centrally, occupying the bulk of the inner portion of the teeth. ${ }^{3,4}$ The pulp normally is not mineralized, except when pulp stones (pulpolithes) are formed in pathological conditions, and/ or when diffuse mineralizations fill the pulp cavity. Pulp horns are persisting structures, even during aging. The pulp comprises a central pulp chamber in the crown of the teeth, and in the roots, the pulp is located in radicular canals.

Depending on the aging phenomenon, the pulp is gradually reduced in volume, and vary according to the species. In rats, the coronal pulp chamber progress at greater rate on the roof compared to the floor. It is slowly reduced in size. The thickness of dentin located in the interradicular dentin increased significantly occluso-gingivally. From available data, it was concluded that

a. no significant change occurrs in the dentin located at the roof of the pulp chamber,

b. lining the floor of the pulp chamber a definite increase in thickness formation is responsible for the reduction in size,

c. the walls of dentin are reduced in size within the pulp chamber. However it should be noted that the horns remain unfilled despite secondary dentin formation,

d. the height and width of the pulp chamber showed a decrease with advancing age. ${ }^{5}$

On the opposite, in humans regular secondary dentine is deposited in larger amounts on the floor compared to the roof. It has also been specified that greater deposition is often seen on the floor, ${ }^{6}$ although some data suggested that age has a influence on secondary dentine formation. ${ }^{7}$ In the crown and namely in predentine, collagen fibers are rather thick, more densely packed and often parallel to the odontoblast processes. Coronal odontoblasts are columnar, whereas in the root, odontoblasts are cuboidal. ${ }^{8}$ The composition in phosphoprotein differs between crown and root odontoblasts, the root dentin containing only 
half the amount of dentin phosphophoryn present in the crown. ${ }^{9}$ In addition, differences have also been reported in. ${ }^{10}$

Four functions of the pulp have been actually identifed: nutritive, protecting/sensory, defensive/reparative and formative. The pulp is an heterogenous tissue, and the expression of STRO- 1 was evaluated to be less than $2 \%$ of the total pulp cell population. CD146 labeled cells are located within dental pulp.

\section{The healthy dental pulp: the cells}

Goldberg \& Lasfargues ${ }^{11}$ have put emphasis on the substantial differences found between the dentin composition and the proteins located within the dental pulp. This cast doubt on the notion of a pulpo-dentinal complex, each of these structures having his own composition. In the dental pulp, odontoblasts (and/or odontocytes), and fibroblasts (pulpoblasts) have been identified, as well as undifferentiated mesenchymal cells, macrophages, dendritic cells and lymphocytes, plasma cells, nerve fibers. All these cells are recognized within the pulp. Mast cells, and fat cells are included in a ground substance, composeed by collagens, elastic and reticular fibers, glycosaminoglycans and proteoglycans, fibronectin, metalloproteinases (MMPs) (collagenases, and tissue inhibitors of MMPs), lipids (phospholipids and proteolipids). By contrast, dentin is mostly composed of type I collagen, and non-collagenous proteins implicated in mineralization. The presence of enzymes specifically associated to pulp cell functions has also been established. ${ }^{12}$

Dentinal tubules are invaded by oral bacteria. This is followed by the colonization and a subsequent infection of the root canal system. ${ }^{13}$ Therefore, although streptococci and Actinomyces are major components of dental plaque, they may initiate tubule and pulpal infection. Streptococci are the primary bacterial colonizers, and adhesion of streptococci to the acquired pellicle is an essential first step in colonization of the tooth.

The number of dentinal tubules permm ${ }^{2}$ varies from 15,000 in the outer dentin to 45,000 at the dentin border. Large tubules are seen near the pulp (for diameter approximately $2.5 \mu \mathrm{m}$ in width). In the outer part of dentin, near the cemento-enamel junction the diameter of the tubules is reduced to about $0.9 \mu \mathrm{m}$. The mean diameter of the tubules vary between 2.85 and 3.10 micrometers. Tubule density in the middle layer was higher in bovine root dentin. According to Schilke et al. ${ }^{14}$ about 18.243+/-3.845 tubules are scored in human deciduous dentin vs $23.738+/-4.457$ in human permanent dentin.

\section{The two peripheral sub-odontoblastic pulp layers and the central pulp}

Beneath the dentin and the continuous layer of odontoblasts, two outer pulp zones have been identified. They include:

The cell free zone: also named the Weil's zone (40 micrometers wide) containing blood vessels, unmyelinated nerves, and macrophages. Mast cells (present only during pulpal inflammation) display round nucleus and dark staining granules. This layer is located peripherically. Plasma cells, lymphocytes, neutrophils, eosinophils, basophils have also been identified in this area.

The cell rich zone: contains fibroblasts and undifferentiated mesenchymal cells. In addition, it includes macrophages, dendritic cells and lymphocytes. Mesenchymal cells are distributed throughout the pulp, especially around perivascular area. They are acting as totipotent cells. In older teeth, the cell number and ability to differentiate comes down.
In the central part of the pulp: fibroblasts and immunocompetent cells were identified as T-lymphocytes, B-lymphocytes, dendritic cells, NK cells. In human deciduous teeth, HLA-DR+, CD68+, and factor XIIIa are present, whereas T and B-lymphocytes form clusters. ${ }^{15}$

The nuclear factor 1C (NFIC) is determining the root formation, but has no influence on the crown. Osterix is strongly decreased in the Nfic-KO tooth root, namely in the expression of DMP-1 and DSPP. This genetic mutation leads to short roots, and a thin dentin, with few dentin tubules. ${ }^{16}$

\section{Blood and lymph vascularization}

Arterioles have thicker walls than venules. Vascular cells surround the lumen. They constitute three different layers : the intima formed by endothelial cells, the media which includes smooth muscle cells and elastic fibers, and the adventice, a thinner layer implying the association of fibroblasts and a extracellular matrix. Veins display thin walls, collagenous and smooth fibers. Arterioles enters in the pulp via apical and accessory foramina. The primary feeding arterioles divide into secondary and terminal arterioles. Pre-capillaries are in continuity with capillaries. They form loops of terminal capillaries and vascularize areas of about 100 to $150 \mu \mathrm{m}$. Following post-capillaries, collecting veinules merge into veinules and exit from the pulp via the apex. Capillaries are surrounded by a continuous basement membrane. Endothelial cells are fenestrated, and/or associated with peri-endothelial cells which contain Weibel-Palade bodies. Thick wall thickness characterizes arteries, whereas thin wall thickness is associated to veins. Endothelial cells are linked by desmosomes and gap-junctions, forming tubular structures. Trans-cellular pinocytotic vesicles are absorbed near the lumen of the capillaries, and transfered to the outer extracellular border through the cytoplasm of endothelial cells. The content of these vesicles is released in the amorphous ground substance located in the adventice of arterioles, in large spaces between capillaries, connecting the network of the pulp extracellular domains. Vasculogenesis is under the control of vascular endothelial growth factor VEGF and VEG receptors (VEGFR), FGF, angiopoietin 1 and 2, placental growth factors and angiogenin receptors. Endothelial fenestrations are important with respect to odontoblast nutrition at the periphery of the pulp. ${ }^{17}$ Thus, in the dental pulp, the levels of alkaline phosphatase, bone sialoprotein and dentin sialoprotein are increased. In the root, capillaries display a continuous fisher-like arrangement, in contrast with the loops seen in the coronal pulp. Intercellular junctions of the desmosome and gap-like type regulate the pulpal circulation (vasoconstriction and vasodilatation), according to Takahashi. ${ }^{18}$

Lymph vascularization is regulated by vascular endothelial growth factors (VEGF-C and D) and their receptors (VEGFR-3). Lymph capillaries are missing in the coronal and middle regions of the pulp, but extensively present in the apical region. Lymph collected in interstitial tissue is drained toward the apex via lymph capillaries. The lymph captured by unsealed endothelial cells flows in the apical direction toward the lymph node chains located in the neck. Intercellular junctions are of the gap type, with discontinuities in the basement membrane.

\section{Differentiation of pulp cells into neuronal precursors}

Contributing to the pulp innervation:

1. A neural crest-derived cell population, expressing the lowaffinity nerve and growth factor receptor (LANGFR).

2. A mesenchymal cell population, expressing the $\beta 1$-integrin receptor subunit. ${ }^{19}$ 
3. Both have the ability to differentiate into osteoblasts, adipocytes, chondrocytes, smooth muscle cells and neuronal precursors. ${ }^{20}$

Nerve fibres involved in pain transmission include A-alpha myelinated fibers (13-20 $\mu \mathrm{m}$ diameter), A-beta fibres (large 5-12 $\mu \mathrm{m}$, myelinated, fast conducting), A-gamma (myelinated , 3-6 $\mu \mathrm{m}$ ) and A-delta fibres (small, lightly myelinated, providing a slow conducting answer, with sharp sensation of pain), B-fibres (myelinated) and C fibres (small and unmyelinated) They are slow conducting pain (mechanical, thermal, and chemical pain, providing a response to all types of noxious stimuli). ${ }^{21} \mathrm{~A}$-delta and $\mathrm{C}$-fibres are nociceptive, whereas A-beta and A-alpha are mechanoceptive. ${ }^{21-25}$ Molar root pulp lacked Fos/Fra-IR. ${ }^{25}$

Innervation of human dentin was reviwed by many authors. Silver impregnated fibres were noted in the pulp and dentin as well. Intratubular nerve fibrils have an approximate diameter of $0.2 \mu \mathrm{m}$ with 1-30 intratubular fibrils/ 2000-2800 tubules. ${ }^{26}$ The hydrodynamics of dentinal fluids theory has still an impact on the understanding of the pulp nerve physiology. ${ }^{27}$

The presence of nerve fibers immunoreactive to protein gene product 9.5, calcitonin gene-related peptide (CGRP), substance P and neuropeptide $\mathrm{Y}$ was established. More immunoreactive nerves were CGRP positive. ${ }^{28,29}$ The protein expression patterns of early (Nestin, PSANCAM), intermediate (-III tubulin), and late (NF-M or NF-H) neuronal-associated markers expressed by DPSCs cultured in the different neuronal inductive conditions, were determined by immunocytochemical analysis. Following neural induction in vitro, DPSCs were shown to constitutively express the immature markers nestin and PSANCAM, which are associated with neuronal precursors and immature neuronal-committed progenitors through to neuroblasts, respectively. ${ }^{30}$ Surface molecules characterization were used together with cytometric analysis. The surface markers to SCAP includes STRO-1, ALP, CD24, CD29, CD73, CD90, CD105, CD106, CD146, CD166 and ALP, Oct4, Nanog, beta 2 integrin positive, stage-specific embryonic antigens (SSEA-3, SSEA-4), and tumor recognition antigens (TRA-1-60 and TRA-1-81). They were negative for CD14, CD34, CD45, CD18, CD150 and HLA-DR. ${ }^{31}$ Calcitonin gene-related peptide (CGRP) was recognized in the rat molars. ${ }^{32}$ Nerve growth factor (NGF), glial cell line-derived neutrophilic factor, vasoactive intestinal peptide (VIP), neuropeptide Y (NPY) and somatostatin were found in the carious pulps versus non-carious teeth. The most studied TRP channels receptors (TRPV1) are required for heat detection, low $\mathrm{pH}$, and capsaicin causing a warm or burning sensation. TRPV2 receptors were described as heat response and highly expressed in the neurons innervating the dental pulp. The TRPA1 receptor is activated by cold temperature. Bradykinin modulates the TRPA1 receptor activity. Voltage-gated sodium and potassium channels are characterized as either tetrodotoxin resistants (TTX-R) or sensitive (TTX-S). Autonomic innervation belongs to the sympathetic division of the autonomic system. Sympathetic stimulation provides effective vasoconstriction. ${ }^{33,34}$

Dense innervation of human radicular dental pulp was revealed by immunocytochemistry for protein gene-product $9.5 .^{35}$ The gliaspecific S-100, neurofilament protein, neuron-specific enolase was also identified in incisor. ${ }^{35-37}$ The hydrodynamic effects of fluid displacement within the dentinal tubules and/or the odontoblast processes. They may activate mechanoreceptors of sensory nerve axons. Odontoblasts are also implicated in the regulation of pulp blood flow and in pulp inflammation. ${ }^{38}$

\section{Degradation of the exogenous pellicle associated with the pulp tissue and plaque bacteria}

Acquired pellicle formation is due to salivary glycoproteins. It includes statherin, proline-rich peptides and host defence components, implicated in reversible and permanent attachment. The initial colonizers are mainly streptococci (S. sanguinaris, S. oralis and S. mitis). Once colonized, the attached population form a confluent layer. Maturation of the biofilm include the synthesis of extracellular matrix of polymers which includes soluble and insoluble glucans, fructans, proteins and extracellular DNA synthetized by glucosyltransferases and fructosyltransferases. A small sample of plaque may contain up to 100 distinct species, S. mutans having properties to act as cariogenic bacteria. ${ }^{39,40}$

\section{Degradation of the dental pulp due to degradation by endogeneous enzyme}

MMPs can be divided into 5 groups: collagenases (MMP-1, -8 , $-13,18$ ), gelatinases (MMP-2 and MMP-9), stromelysins (MMP -3, $-7,-10,-11,-12$ ), membrane-type MMPs (MT-MMPs) (MMP-14, $-15,-16,-17$ ) and MMP-20 (also called enamelysin) implicated in the degradeation of amelogenins. ${ }^{41,42}$ Carious lesions imply the degradation of collagens and other extracellular matix components. Gelatinase A (MMP-2) and gelatinase B (MMP-9) are cuting the collagen fibers into two parts (1/4 th and $3 / 4$ th). MMP2 remove telopeptides from the ends of helical collagens. Only MMP-8 can cleave the collagen fibres into two large fragments. Cathepsin K splits both helical and telopeptide regions. Cathepsins $\mathrm{B}$ and $\mathrm{L}$ cleave the non-helical telopeptides estensions of collagens, whereas cathepsin $\mathrm{K}$ cleaves the collagen at the triple helical region. Lysosomal cystein cathepsins degrade type I collagen, laminin, fibronectin and proteoglycans. ${ }^{43,44} \mathrm{MMP}-2$ is an uppregulator of tissue inhibitor of MMP-2 (TIMP-2), acting on the membrane type-I matrix metalloproteinase (MT1-MMP). It activates the tartrate-resistant acid phosphatase, an enzyme implicated in dentin resorption. Cysteine cathepsin proteinases modulate tertiary dentinogenesis by release and activation of latent TGF- $\beta$ or other growth factors. The TGF- $\beta$ signaling pathway namely SMAD-2 and SMAD-4 explain the increased synthesis of collagen in caries. All theses MMPs and their inhibitors are involved in the formation of reparative dentin. ${ }^{45}$ Spontaneous degradation of the pulp tissue occurs in the presence of endogenous proteinases and/or cariogenic bacteria. Inflammatory and/or immunocompetent pulp cells regulate the degradation process of the dental pulp.

\section{Inflammatory and immunocompetent pulp cells}

Inflammatory cells include pulpal dendritic cells, histiocytes/ macrophages and T-lymphocytes.

Lymphocytes are classified as B- or T- lymphocytes. T-lymphocytes involve cells, such as helper/inducer T-cells (CD4+) and cytotoxic/ suppressor T-cell (CD8+). The B-lymphocytes are rare or lacking in the normal (healthy) pulp. During an early inflammatory reaction phase, B-lymphocytes are present, but they are increased in advanced phases. In contrast, the T-lymphocytes are revealed by immunostaining both in normal and inflammed pulps. Lymphocytes are found as normal resident cells.

The dendritic cell family migrates from the bone marrow to the pulp. Macrophages are scavengers cells displaying phagocytic capacities. Dendritic cells (DC) are concentrated at the periphery of the coronal pulp. They are members of the class I and II major histocompatibility complex (MHC). 
Resident macrophages appears at perivascular locations. Nonresident macrophages and monocytes constitute minor macrophage populations. Mast cells containing histamine, leukotrienes and palatet-activating factors are implicated in immediate hypersensitivity reaction.

Melanocytes and dental pulp cells arise from the neural crest cell population. Melanocytes are elongated dendritic cells with specific organelles, called melanosomes, which contain all components required for melanin biosynthesis. The main melanosomal proteins involved as catalytic and/or structural components in melanin biosynthesis include tyrosinase (TYR), the tyrosinase-related proteins-1 (TRP- 1) and tyrosinase-related protein-2 (TRP-2). MART1 (Melanoma Antigen Recognized by T lymphocytes) is required for melanosome structure and maturation. Polymorphonuclear leukocytes are mostly involved in the capture and degradation of bacteria, namely caries-associated bacteria invading the dental pulp. ${ }^{45}$

Dental pulp stem cells have a great potential in regenerative medicine. ${ }^{46-48}$ They may differentiate into several different types of cells: osteoblasts, adipocytes, chondrocytes, odontoblasts, neurons, and melanocytes. Studies show that DPSCs spontaneously differentiate in vitro toward the melanocytic lineage; these cells show morphological and molecular features of melanocytes; DPSCs cultured in MEM differentiate into melanocytes at considerably slower rate than those cultured in DMEM. ${ }^{49}$ Immune defense mechanisms have been analyzed. ${ }^{50}$ B-lymphocytes produce antibodies against specific antigens following blast formation, proliferation, and maturation into plasma cells. T-lymphocytes are divided into T-helper cells (CD4+) and cytotoxic T-cells (CD8+). When CD4+ is activated, T-lymphocytes are identified according their recognition of membrane-bound T-cell receptors. $\mathrm{CD}^{+} \mathrm{T}$-lymphocytes are further classified into Th1 and Th2 cells. Th1 produce interleukine (IL-2) and interferon-gamma (IFN $\gamma$ ). Undifferentiated cells participate in dentin and pulp regeneration. The expression of DSPP, Cbfa1, and collagen types I and III in pulp is currently considered: DSPP is dentin-specific and expressed mostly by odontoblast cells. ${ }^{51}$ The transcriptional factor Cbfa1 is expressed in mouse dental pulp clonal cell lines. ${ }^{52}$ Both type I and III collagen are produced by pulp fibroblasts. Whereas collagens type III, V, VI have been also identified in the pulp, the dentin collagen is almost exclusively of the type $\mathrm{I} .{ }^{12}$

Antigen-presenting cells (APC) : The Major Histocompatibility Complex (MHC) region encodes transplantation class I and class II molecules. Dendritic cells, macrophages, B-lymphocytes, endothelial cells, and various epithelial and mesenchymal cells are constitutive or inducible cells expressing Class II MHC molecules. T cells, helper/inducer $\mathrm{T}$ cells, cytotoxic/suppressor $\mathrm{T}$ cells, macrophages, $\mathrm{B}$ cells, and Class II antigen-expressing cells are located at the pulp periphery. Dendritic cells expressing Class I antigens (HLA-DR, -DQ), indicating a capacity for presentation of antigen to $T$ helper cells, are located mostly in the odontoblastic layer. They are also found in the central portions of the pulp. T-lymphocytes, divided into helper/inducer and cytotoxic/suppressor cells, are observed in all pulp specimens, whereas B cells are undetectable in most of the healthy pulp samples examined..$^{53}$

Dendritic cells (DC) have a dendritic morphology, high motility and limited phagocytic activity. Macrophages are derived from the same myeloid precursor cells in the presence of macrophage colonystimulating factor (M-CSF). Two types of DC and macrophages expressing class II antigens have been identified in the normal rat incisor pulp: one with a pronounced dendritic appearance, located at the periphery of the pulp, and the other with morphologic characteristics similar to macrophages, established in the central part of the pulp. ${ }^{54}$ Double immunohistochemistry using anti-OPN and class II major histocompatibility complex (MHC) antibodies was performed to determine the relationship between immunocompetent cells such as dendritic cells and macrophages with the expression of OPN. They are DC and Langerhans cells in peripheral nonlymphoid tissues. DC and macrophages originate both in the bone marrow. Macrophages act as scavenger cells. They have prominent phagocytic capacity. T-lymphocytes are essential normal residents of the dental pulp. CD4+ and CD8+ T-lymphocytes are present in normal pulp. Resident macrophages (histiocytes) are identified in healthy dental pulp. They are located in perivascular regions of the outer pulp. Minor populations include ED1+/ED2-/OX6+ cells (class II+ non-resident macrophages) and EDI+/ED2-/OX6- cells (class II- monocytes). Several types of immunocompetent cells that are considered to be essential for the induction of antigen-specific reactions in the dental pulp. Class II molecule-expressing pulpal DCs are of primary importance..$^{54}$ As far as the structural arrangement of the immunocompetent cells is concerned, the dental pulp is not unique but shares several characteristics with other connective tissues. There is indeed some evidence showing that pulpal DCs are actually able to respond to transdentinal antigen provocation, and that the kinetics of these cells are strongly influenced by changes in dentin permeability.

\section{Apoptosis of pulp cells}

In addition to metalloproteinases, the disintégrin and metalloproteinase 28 (ADAM28) was identified in the dental pulp. Disintegrin and metalloprotease (ADAM) proteins are a family of membrane-anchored glycoproteins implicated in development, and protein ectodomain shedding. ADAM28 genes were detected from patients with congenital hypoplasia of tooth root, and the relationship between ADAM28 and tooth development was identified. The expression and localisation of ADAM28 were detected in tooth germ and dental mesenchymal cells. ADAM28 is expressed at each stage of tooth germ development, in human dental follicle cells, in papilla cells, pulp stem cells, and human dental cervical loop epithelial cells. ADAM28 participates in the regulation of odontogenic mesenchymal cells through progressive reciprocal induction. ${ }^{55}$ Zhao et al. ${ }^{56}$ investigated the influence of ADAM28 on the proliferation, differentiation, and apoptosis of human dental pulp stem cells. Investigations were carried out to determine the effects of ADAM28 on HDPSCs expressing dentin sialophosphoprotein (DSPP), dentin matrix protein (DMP1), and bone sialoprotein (BSP). The proliferation was inhibited (negative effect), and specific differentiation of HDPSCs was promoted. The expression of DSPP was enhanced.

During development, apoptosis eliminate unnecessary cells and vestigial structures. Apoptosis plays a keyrole in tooth shape and size. ${ }^{57}$ Macrophages and MHC class II-expressing dendritic cells eliminate apoptotic fragments. ${ }^{58}$ Apoptosis includes the active participation of Fas ligands (Fas), tumour necrosis factor (TNF), nerve growth factor (NGF), nitric oxide (NO), lipopolysaccharide (LPS), host immune reactions, kinins and glucocorticoids. The inactive form of procaspases is activated into the protease caspase, eventually cleaving the host cell by acting on a variety of cell structures such as the nuclear membrane. The cell shrinks and there is a loss of cell-cell junctions resulting in detachment from adjacent cells. The chromatin condenses, the cytoplasm 'blebs' forming'pseudopods', and the cell breaks up into fragments known as 'apoptotic bodies'. Indirectly activated endonucleases lead to breakdown of the DNA 
intomultiples of 180-200 base pair fragments. Finally, macrophages or adjacent cells phagocytose the apoptotic bodies. There is no loss of membrane integrity. It is a non-inflammatory process, ending by nuclear fragmentation. ${ }^{59}$

Notch proteins are important regulators of stem cell function, with capacities to induce proliferation and/or differentiation. Caspase- 9 dependent pathway is also an inductor of apoptosis in human dental pulp cells. ${ }^{60}$ Dental injuries often lead to odontoblast apoptosis, which triggers activation of dental pulp stem cells followed by their proliferation, migration, and differentiation into odontoblast-like cells, elaborating a reparative dentin. ${ }^{61}$ Fas ligand (FasL), a transmembrane protein, plays an important role in inducing the Fas apoptotic pathway. ${ }^{62}$ Proteinase-activated receptors (PARs) are G proteincoupled receptors that undergo irreversible proteolytic activation by proteases. Activation occurs via the proteolytic cleavage at the $\mathrm{N}$-terminus exodomains, revealing a new $\mathrm{N}$-terminal domain that binds to the receptor, and initiate signaling. The activation of PARs promotes coupling to heterotrimeric $\mathrm{G}$ proteins and subsequent signal regulatory mechanism at the plasma membrane. Four members of the PAR family (PAR-1 to PAR-4) have been described. ${ }^{63}$ Odontoblasts orchestrate the pulpal inflammatory response by producing a variety of cytokines and chemokines such as tumor necrosis factor (TNF)- $\alpha$, interleukin (IL)-8, TLR2, and TLR4. The stimulation of PAR promoted a significant increase in the gene expression of MMP2, MMP-9, MMP-13, and MMP-14. Human dental-pulp complex constitutively expressed PAR-1 and PAR-2 in the odontoblastic region. Immunostaining of the dental-pulp complex region showed that labeling was 1.9-fold more intensive for PAR-2 compared to PAR1. The results presented by Alvarez et al. ${ }^{63}$ support the hypothesis of activation of upregulated PAR-1 and PAR-2 by endogenous proteases abundant during the inflammatory response in dentin-pulp complex.

Neurotrophin receptor-mediated melanoma antigen-encoding gene homology (NRAGE), is a regulator of apoptosis. ${ }^{64} \mathrm{NRAGE}$ is involved in cellular functions such as cell proliferation, cell motility, cell cycle arrest and apoptosis. The biological effects of NRAGE on the MDPC23 were detected in knockdown of NRAGE that inhibited apoptosis of hDPCs and MDPC-23 through the NF-kB signaling pathway. It has been shown by Saewong et al., ${ }^{65}$ that simvastatin, an inhibitor uses as cholesterol-lowering agent, disrupts the actin cytoskeleton, and decreases the viability of pulp cells probably by inducing apoptosis. It influences the synthetic cascade producing cholesterol and isoprenoid lipid metabolites. Cholesterol plays a crucial role in the organization, lateral heterogeneity, and dynamics of plasma membrane. The level of cellular cholesterol determines functional compartmentalization of membrane lipids and proteins into ordered microdomains (lipid rafts), which may serve as scaffolds for signaling complexes. Proapoptotic effects of statins inhibit isoprenoid synthesis. In conclusion, apoptosis is a key-factor, regulating the non-infected dental pulps, and involved in pulp colonisation and degradation by carious bacteria.

\section{Healthy pulp composition}

\section{Extracellular matrix components of the dental pulp}

Cells and components of the extracellular matrix comprize stuctural fibers (collagen type I and III, fibronectin, elastin) and an adhesive ground substance (fibronectin and laminin), glycosaminoglycans (HS, DS, CS), and more complex proteoglycans (decorin, biglycan, versican), Lipids, phospholipids and proteolipids, Cbfa 1 and Cbfa 3, have also been identifed within the dental pulp.
Fibronectin is an adhesion glycoprotein of the extracellular matrix, exists as a dimer, that displats a molecular mass of $\sim 550 \mathrm{kDa}$. Multiple domains of fibronectin show binding affinities for collagen, fibrin, heparin, and specific cell membrane receptors such as integrins.

Root-analogue versus crown-analogue radioautographic investigations reflect extensive differences between the two dentin portions. The processing of collagen incorporation from the predentin into dentin is faster in the root aspect. ${ }^{66}$ Small Integrin Binding Ligand N-linked Glycoproteins (SIBLINGs), are cleaved into DSP and DPP, DMP1, BSP, osteopontin, and matrix extracellular phosphoglycoprotein (MEPE). The SIBLING molecules can form specific complexes with different MMPs, in either their latent or active forms. In salivary glands as well as in kidney, BSP is able to bind specifically to MMP-2, OPN to MMP-3, and DMP-1 to MMP9. ${ }^{67-69}$ Therefore, MMPs may be enzymatically active in areas with high SIBLING concentrations, and promote tissue remodeling. ${ }^{70}$ Osteocalcin, osteonectin (or SPARC protein, also named BM40), tenascin and fibronectin are members of the non-phosphorylated ECM family. In addition, dentin contains weakly phosphorylated matrix molecules, such as amelogenin and some proteoglycans, and non-phosphorylated proteins as well. While osteonectin may contribute to the mineralization process, osteocalcin and the matrix Gla protein serve as nucleator inhibitors. The small leucine-rich proteoglycans (SLRPs), (decorin, biglycan, fibromodulin, lumican, and osteoadherin) have been identified in dentin. Although they are not specific to dentin and can be found in many other mineralized or non-mineralized tissues, they have been implicated in dentin calcification. They are involved in the transport of collagen fibrils through the predentin and in collagen fibrillation. ${ }^{71,72}$ Dentin was also shown to contain several growth factors, such as the transforming growth factor beta 1 (TGF- $\beta 1$ ), basic fibroblast growth factor (FGF2), and insulin-like growth factors I and II (ILGF I and II). Other components phospholipids and serum-derived proteins, such as albumin and $\alpha_{2} \mathrm{HS}$ glycoprotein represent potential substrates for degradation by proteinases. Indeed, the localization of several MMPs in dentin is consistent with the notion that these enzymes participate in the degradative process associated with caries development. ${ }^{70}$

Subsets of cells expressing markers of bone (alkaline phosphatase, osteopontin, and bone sialoprotein), smooth muscle ( $\alpha$-smooth muscle actin), and endothelial cells (MUC-18) are represented in dental pulp stem cells (DPSCs) and bone marrow stem cells (BMSCs). The heterogeneous nature of DPSCs may reflect differences in their developmental stages or represent different pulp cell lineages. The postnatal dental pulp contains cells that are clonogenic, highly proliferative, and capable of regenerating a tissue, properties that effectively define them as stem cells.

Genes (dentin sialophosphoprotein (DSPP), gene expression and gene receptors (enamelysin/matrix metalloproteinase 20), growth factors, and growth factor receptors (Smad1, Smad7, BMP2 and BMP-4, Phex (phosphate-regulating gene with homologies to endopeptidases on X-chromosome), enzymes or extracellular proteins are associated in the dental pulp. Growth factors regulate the proliferation and differentiation of odontoblast precursors include the basic fibroblast growth factor, platelet-derived growth factor, epidermal growth factor, insulin-like growth factor I, tumor necrosis factor $-\alpha$, and IL- $\beta 1$.

The roles of IL- $1 \alpha$ and TNF- $\alpha$ in early pulp inflammation was investigated by determining the alkaline phosphatase activity, 
osteonectin, osteocalcin, bone sialoprotein and heme oxygenase-1 expression (HO-1). IL-1 $\alpha$ induces ALP, ON and OC in tooth mineralization and plays a role in the cytoprotection of pulp cells via H0-1 expression. Long-term treatment of the pulp by TNF- $\alpha$ may inhibit tooth mineralization. ${ }^{73}$ Runx3, osterix, DSPP, enamelysin, KLK4, alkaline phosphatase, BSP are also extracellular components. Matrilin-2 and -4, CD44 and interleukin-1 $\beta \alpha \rho \varepsilon$ linked to cellular and pericellular molecules. Pulp stem cells express bone markers such as bone sialoprotein, alkaline phosphatase, type I collagen and osteocalcin. Early markers of pulp stem cells, and cytokines are also found in the dental pulp. ${ }^{69}$ Basic fibroblast growth factor (bFGF) is involved in the development and repair of dentin and pulp. Pulp cells produce SPARC (secreted protein, acidic and rich in cysteine), alkaline phosphatase (ALPase) and calcification proteins. SPARC are 30 - to $43 \mathrm{kDa}$ molecules bound to odontoblasts, but absent in pulp cells. However, SPARC is present both in the odontoblast layer and in predentin. ${ }^{74} \mathrm{~A}$ few metalloproteinases have been identified in dental tissues, namely collagenase-1 (MMP-1), collagenase-2 (MMP-8, human neutrophil collagenase), and collagenase 3 (MMP13). Collagenases and collagenase inhibitors present in the dental pulp (gelatinase A (MMP-2) and gelatinase B (MMP-9), enamelysin (MMP20) are localized in the extracellular matrix of the dental pulp. TGF of this growth factor of note, MMP-8, expressed by odontoblasts and pulpal cells, is of the mesenchymal-type MMP-8. A direct effect of substance $\mathrm{P}$ and calcitonin gene-related peptide was evidenced in vitro on fibroblast-like cells derived from human dental pulp. They showed concentration-dependent stimulation of cell proliferation. A direct effect on pulp cells is consistent with a role of the neuropeptides in pulp healing, regulating the level of cell proliferation, rather than having a functional activity. ${ }^{75}$ Insulin growth factor1 (IGF) and insulin-like growth factor binding protein-2 and -3 (IGFBP-2 and -3) regulate matrix mineralization of differentiating human dental pulp cells.

\section{Non-carious and carious dental lesions}

\section{Non carious destructive processes. ${ }^{76}$}

Abrasion describes the weaning way of a structure through mechanical processes such as grinding. It can be localized or diffused. They include brushing, the time spend and force applied, the $\mathrm{pH}$ and amount of toothpaste on the brush.

Demastication is influenced by the abrasiveness of the individual food.

Attrition results of tooth-to-tooth contact with no foreign substance intervening. The individual degree of attrition is associated with age.

Abfraction is used to describe a wedge-shaped defect at the cement-enamel junction.

Resorption describe the process of biological degradation. Dental resorption describe the biological removal of dental hard tissue by cementoclastic, dentinoclastic and ameloclastic activities.

Erosion describe the gradual destruction of a surface by electrolytic or chemical processes. Chemical etching by an acid or chelator may occurr without bacterial involvement. This is the case in anorexria nervosa or bulimia.

\section{Carious decay}

Dental caries is the localised destruction of dental hard tissues by acidic by-products from bacterial fermentation of dietary carbohydrates. ${ }^{77}$ All types of microorganisms in the oral cavity are not equally able to ferment carbohydrates. It is logical to look for major caries pathogens. However, in patients with excellent oral hygiene there is virtually no 'plaque' on the tooth surfaces and therefore S. Mutans may be below the detection level. This should be combined with the fact that streptococci are not primary colonisers of tooth surfaces. Relatively high proportions of Streptococcus Mutans may persist on tooth surfaces without caries progression while caries may develop also in the absence of these species. Therefore, there is evidence that caries is not a classical infectious disease. ${ }^{78}$ Endogenous bacteria, largely Streptococcus mutans, Streptococcus sobrinus ; and Lactobacillus produce weak organic acids as a by-product of metabolism of fermentable carbohydrates. This acid causes local $\mathrm{pH}$ values to fall below a critical value resulting in demineralisation of tooth tissues. This process leads either to cavitation within the tooth or to the repair and reversal of the lesion, or to the maintenance of the status quo. Remineralisation is frequent, especially when the biofilm $\mathrm{pH}$ is restored by saliva, acting as a buffer. The acids produced include lactic, acetic, formic and propionic, all of which dissolve the mineral of the enamel and dentine. ${ }^{79}$ Many of the functions of the salivary proteins have been established together with the very important protective role of saliva in the reversal or arrestment of the caries process. Dental caries is a disease that is manifested as a dynamic process in the mouth. In the crown and carious root dentin, streptococcus are identified (namely S.mutans, S. sobrinus, S. intermedius, S. morbillorum and S. sanguinis), as well as a group of actinomyces (A. israelii, naeslundi, viscosus, and odontolyticus bacteria). More than 300 bacterial species are components of the oral microflora. However, only few species are able to invade and infect the root canal space. Pioneering studies demonstrated that, in addition to streptococci, lactobacilli, and Actinomyces, obligately anaerobic species such as Fusobacterium, Peptostreptococcus, Eubacterium, Propionibacterium, Veillonella, Wolinella, Prevotella, and Porphyromonas dominated the root canal microflora.

Studies on the microbiology of primary dental caries in human indicate:

a. The role of the streptococci mutans in the initiation of caries of smooth surfaces and fissures of crowns, and suggests their potent role in induction of root surface caries.

b. That lactobacilli are implicated as important contributory bacteria in tooth decay.

However, their role in induction of lesions is not well supported, despite the major roles played by the Streptococcus mutans and the lactobacilli. ${ }^{80}$ Molecular/genetic studies of implicated bacteria isolated from humans, indicate that mutans streptococci spread vertically, mostly from mothers to their children. ${ }^{81}$ Mutant streptococci, Sanguinis/other streptococci, Enterococci, Lactobacilli and Actinomycetes are the main bacterial groups favoring the development of dental caries. The tooth surface selectively adsorbs various acidic glycoproteins (mucins) from the saliva, contributing to the formation of the acquired enamel pellicle (AEP), which varies in thickness from 0.1 to $3 \mu \mathrm{m}$, and contains a high number of sulfate and carboxyl groups. The initial colonization of the AEP involving Actinomyces viscosus or A. naeslundii with $\mathrm{S}$. sanguis have been described. Streptococci are found in plaque. The fermentation of mannitol and sorbitol, produce extracellular glucans from sucrose, and they are cariogenic in animal models, with the exception of S. ferus. Data provide evidences that S. mutans (possibly S. sobrinus, and 
lactobacilli) are human odontopathogens. As such, dental caries is a diagnosable and treatable infection. Other species such as S. sobrinus appear to be important primarily in smooth-surface decay and may be a cariogenic determinant when rampant decay occurs. Colonization by S. mutans occurs after tooth eruption, and if the fissures become colonized in their depths, the decay may be inevitable. However, if this colonization is delayed until the fissure depths are occupied by other bacteria, the carious decay will not occur or its occurrence will be greatly reduced. This understanding of the ecology of S. mutans suggests treatment strategies. They may have profound effect on the incidence of dental decay in human populations. The mineralized dental tissues are the relevant oral solid surfaces, and these surfaces are coated by a pellicle to which the microbial cells attach. The colonizers generate a matrix of exopolymer within which cells grow. The role of the biofilm in driving the caries process has some important clinical implications.

\section{Enamel and dentin carious lesions}

\section{Early enamel caries}

The surface zone appears as a «white-spot lesion » due to a demineralization/reminéralization process. The sub-surface lesion is covered by an apparently intact surface layer, and have lost as much as $50 \%$ of the original mineral content. Magnesium is reduced by $20.1 \%$. The dark zone is hypomineralized, with a loss of $12 \%$ in magnesium, and $6 \%$ reduction in mineral per unit volume. The translucent zone is apparently a hypermineralized structure..$^{45}$ The two most important group of bacteria that produce lactic acid are the S. mutans and lactobacilli. The mineral loss may be stopped or reversed by the gain produced by saliva. In other words, the lesion may be arrested or remineralized, and this can occur at any stage of lesion formation. ${ }^{82}$ The initial carious lesion expand in the direction of the dentinoenamel junction. The enamel decay cross this border and spread along the junction, before penetrating into dentin.

\section{The dentin carious lesion}

Dentin is a vital tissue containing the odontoblast processes. Beneath a layer formed by food debris (vegetal or animal- meet), the soft carious dentin may be removed by excavators. There is no peritubular dentin. The most common defense reaction is the deposition of mineral within the dentinal tubules. Tubular sclerosis within dentin tubules is visible before the advancing front of the carious lesion. It extends toward the enamel-dentin junction and comes in contact with the enamel-dentin junction. A brownish discoloration of the dentin appears, and this is the first sign of dentin demineralization. This appearance is due to the lateral spread of the lesion. The other important defense reaction is the formation of reactionary dentin, beginning before the bacterial invasion of the dentin tubules. Examination through a section of the carious dentin reveals that

1. The upper part of the carious dentin include zone of degradation, where food debris accumulate.

2. A zone of bacterial invasion where soft carious dentin is found This zone can be cleaned by hand excavation, displaying enlarged tubules, and lacking peritubular dentin,

3. Dentin demineralization where a mixture of widely open and closed tubules are seen. Invaded (infected) dentinal tubules displayed intratubular reprecipitations. Peritubular dentin gradually reappears, with a gradient of mineralization (increasing from the superficial part of the decay toward the deep sound dentin). At that stage, infected dentin dentin needs to be drilled by burrs.
4. In the sclerotic dentin layer, precipitations of calcium and phosphate occurr, and remineralizations of apatitic and whitloockite crystals occlude the lumen of tubules.

This is followed by fatty degeneration of odontoblast processes inside the lumens of the tubules. « Sound » tubules are limitting the reactionary (tertiary) dentin. Limitted by a calcio-traumatic line (or border) limitting the dental carious dentin, the sclerotic area of the carious decay constitute the outer limits of an apparently healthy pulp. ${ }^{45}$

\section{The root pulp: spontaneous healing, stem cells and induced pulp regeneration}

\section{Human stem cells}

The pulp root canal regeneration involves five different types of human dental stem/progenitor cells characterized as dental pulp stem cells (DPSCs), stem cells from exfoliated deciduous teeth (SHED), periodontal ligament stem cells (PDLSCs), stem cells from the apical papilla (SCAP) and progenitors for dental follicle (DFPCs). ${ }^{83}$ These stem cells constitute a reservoir implicated in pulp repair and regeneration of damaged pulp tissues.

Stem cell niche: The DPSC niches in human pulp were found to be localized in the perivascular and perineural sheath regions. These STRO-1+/CD146+DPSCs form a dentin-pulp-like complex in vivo, similar to the multiple colony-derived DPSCs. STRO-1 staining of apical papilla is positive in the peri-vascular region as well as in other regions scattered all over the tissue (Huang 2009).

Positive markers (STRO-1, CD13, CD44, CD24, CD29, CD73, CD90, CD105, CD106, CD146, Oct4, Nanog and $\beta 2$ integrin), and negative markers (CD14, CD34, CD45 and HLA-DR) are used to identify dental stem cells. Subpopulations are reported to be multipotent stem cells, although CD34 is known to be also markers for hematopoietic lineages. Side population cells exist in porcine dental pulp. They exhibit stem cell properties with self-renewal and multipotency for dentinogenesis, chondrogenesis, adipogenesis and neurogenesis. ${ }^{20}$ In a dog animal model, a subfraction of the side population (CD31-/CD146-) was found to regenerate partially the pulp tissue removed in the pulp chamber.

\section{Induced pluripotent stem cells (iPSCs)}

Stem cells are capable of renewing themselves through cell division and differentiate into many different types of cells. The discovery of induced pluripotent stem (iPS) cells offers the option of autologous transplantation..$^{84,85}$

Retroviral introduction of Oct3/4, Sox2, c-Myc and Klf4 induced pluripotent stem cells (iPSC) were silenced in Nanog iPS cells. ${ }^{86,87}$ Adult chimaeras were obtained. ${ }^{88-90}$

Embryonic induced pluripotent stem cells (ESCs), derived from the early embryos, possess two important characteristics: selfrenewal and pluripotency. They may be utilized for curing clinically a number of degenerative and genetic diseases. The recent advances in induced pluripotent stem cell (iPSC) research have clearly shown that differentiated somatic cellscould be reprogrammed into pluripotent state by ectopically expressing a combination of several transcription factors.

\section{Adult induced pluripotent pulp stem cells (iPSCs)}

iPSCs are reprogrammed from human somatic cells through ectopic expression of various transcription factors. Takahashi and Yamanaka ${ }^{86}$ have shown that a set of transcription factors could reprogram somatic 
cells and acquire a pluripotent stem cell state, in era of induced pluripotent stem cells (iPSCs). ${ }^{19,89-91}$ Nanog is initially dispensable but becomes essential for dedifferentiated intermediate states. ${ }^{92}$ Induced pluripotent stem cells (iPSCs) after isolation from multiple niches, found within the pulp of child's primary teeth (deciduous teeth). ${ }^{39} \mathrm{No}$ changes in the morphology, expression of stem cells markers (nestin, vimentin, fibronectin, $\mathrm{SH} 2, \mathrm{SH} 3$ and Oct3/4). Chondrogenic and myogenic differentiation potential were also induced. Multiple stem cell niches were identified in different zones of dental pulp, while STRO-1 protein localization was restricted to perivascular niches. However, it is also obvious that iPS cells have not a stable phenotype, but their phenotype is modified spontaneously. While downregulation of Thy 1 and subsequent upregulation of SSEA-1 occur at early time, Oct4, Sox2, telomerase, and the silent X chromosome marks late events in the reprogramming process

\section{Cells and growth factors implicated in de novo pulp regeneration}

In dogs, autologous pulp stem/progenitor $\left(\mathrm{CD} 105^{+}\right)$cells leads to a complete apical closure in mature tooth. By day 14, regenerated pulp including nerves and vasculature is followed by new dentin formation along the dentinal wall. ${ }^{93}$ Stem/progenitor cells can be isolated, characterized, and seeded onto synthetic scaffolds consisting of poly-D,L-lactide/glycolide. They may be inserted in the tooth, and transplanted into mice inducing complete pulp regeneration. ${ }^{94}$ The granulocyte-colony stimulating factor (G-CSF) induces subsets of dental pulp stem cells and contribute to the formation of groups of mobilized dental pulp stem cells (MDPSCs). ${ }^{95}$ RT-PCR reveals stem cell markers which include Oct4A, Nanog, Sox2 , Rex1, GDF3 , LIN28, Stat3, and CXCR4. MDPSCs CSF exhibited a much higher expression of angiogenic and/or neurotrophic factors (granulocyte-macrophage colony-stimulating factor [GM-CSF], matrix metalloproteinase 3 [MMP3], vascular endothelial growth factor [VEGF], brain-derived neurotrophic factor [BDNF], glial cell derived neurotrophic factor [GDNF], nerve growth factor [NGF], and neurotrophin 3 [NT-3]) compared with a colony-derived DPSCs without isolation. These implantations implies that MDPSCs have higher angiogenic/vasculogenic and neurogenic potential, although both MDPSCs and DPSCs were positively stained with oil red $\mathrm{O}$, showing mRNA expression of the adipogenic markers. Adipocyte Protein $2(\mathrm{aP} 2)$ and peroxisome proliferator-activated receptor 4 (PPAR 4), are indicating adipogenic induction. Twenty eight days after osteogenic induction, the mineralized matrix was stained by alizarin red in both MDPSCs and DPSCs, expressing the osteoblastic marker osteocalcin. Pulp regeneration potential of human MDPSCs was shown in an experimental model of ectopic tooth root transplantation. Pulp-like tissue in the tooth root with a well-organized vasculature system was regenerated 21 days after transplantation of MDPSCs and DPSCs. Masson trichrome staining revealed a fibrous matrix formation. Immunofluorescent staining analysis with BS-1 lectin showed that neovascularization in the regenerated pulp tissue is significantly larger in MDPSCs compared with the transplantation of DPSCs. Regeneration of dental tissues involves the expression of Dspp, enamelysin/MMP20 mRNA. Transplantation of BMP-2 on pulp cells stimulates the differentiation of the cells into odontoblasts, and results in dentin formation..$^{93-98}$

Colombo et al. ${ }^{99}$ have promoted scaffolds involved in the control of inflammation. Presumably, they facilitate regeneration. The resolvins are a group of molecules that were first described by Serhan et al. ${ }^{100,101}$ Resolvins (RvE1 and RvD1), are molecules derived from omega-3 polyinsatured fatty acids. Resolvins act on immune cells, and produce anti-inflammatory actions (e.g., reducing polymorphonuclear leukocyte infiltration and tissue injury). They are implicated in pro-resolving actions (e.g., increasing phagocytosis activity of macrophages). Resolvins are known to act on immune cells and produce anti inflammatory actions (e.g., reducing polymorphonuclear leukocyte infiltration and tissue injury) and pro-resolving actions (e.g., increasing phagocytosis activity of macrophages). ${ }^{102}$ Resolvins may have great efficacy in the treatment of pulpal inflammation, and the preservation or regeneration of a vital dental pulp. Two broad classes of resolvins have been characterized: the E-series, which are derived from eicosapentaenoic acid, and the D-series, which are derived from docosahexaenoic acid. There are several key pathways for the synthesis of the resolvins in vivo, including via aspirin-acetylated cyclooxygenase-2 (COX2). Resolvin E1 (RvE1) has shown efficacy in a dental context. RvE1 down-regulates the nuclear factor kappa B through the ligand-specific receptor Chem R23, which is expressed by a number of cell types, including monocytes/macrophages, neutrophils, dendritic cells, and $\mathrm{T}$ cells. The delivery of RvE1 stimulate regeneration by resolving the initial inflammatory response in diseased pulp, shifting macrophages from their proinflammatory phenotype. This allows the healing of dental tissues. However, the potential of RvE1 to resolve pulpitis and subsequently enhance dental pulp tissue. However, pulp regeneration remains relatively unexplored.

\section{Growth factors and cell carriers implicated in root pulp regeneration}

Cell- free approaches for dental pulp engineering have been investigated by Galler et al. ${ }^{103}$ Combining stem cells with suitable scaffold material transplanted into the root canal may induce the (re) generation of a pulp-like tissue.

Tissue engeenring includes

1. A bioactive scaffold,

2. Growth and differentiation factors released by dentin, and

3. The recruitment of stem cells from resident populations within the pulp or from the periapical region.

Bioactive dentin matrix components are present in the EDTAsoluble fraction of dentin. They include transforming growth factor beta (TGF- $\beta 1$ ) (and their isoforms 1, 2, and 3), basic fibroblast growth factor (bFGF), vascular endothelial growth factor (VEGF), bone morphogenetic proteins (BMPs-7, BMPs belonging to the TGF beta superfamily of proteins), insulin-like growth factor (IGF-1 and -2), platelet-derived growth factor (PDGF), placenta growth factor (PIGF), hepatocyte growth factor (HGF), and epidermal growth factor (EGF). These molecules are efficient even at very low concentrations and influence cell migration, angiogenesis, and cell differentiation. Bioactive scaffols can be modified. Structural changes involve cross-linking mechanisms, the fiber length and viscoelasticity. The resulting materials are biocompatible and biodegradable, due to cellmediated enzymatic cleavage. Cell-free approaches using bioactive scaffolds, and growth factors may regenerate the dental pulp. After cells recruitment, stem pulp cells re-populate the scaffold and adhere, proliferate, differentiate, and eventually form new tissue. Natural or synthetic polymers, extracellular matrix proteins, self-assembling systems, hydrogels, or bioceramics may be used as scaffolds. ${ }^{104}$ Recent approaches aiming to generate dental pulp rely on established materials, such as collagen, polyester, chitosan, and/or hydroxyapatite. Various aspects including vascularization, cell-matrix interactions, growth-factor incorporation, matrix degradation, mineralization, and 
contamination control should be considered for the success of pulp regeneration. Self-assembling peptide hydrogels are example of smart materials that may be modified to create customized matrices.

A number of biomaterials have been used including :

1. Natural polymers (collagen, fibrin, polysaccharides, alginate, dextran, chitosan, cellulose, starch, hyaluronan, glycosaminoglycans and extracellular matrix).

2. Synthetic polymers (polyester, PLA poly(lactic) acid, PGA poly(glycolic acid), PLGA copolymer of PLA and PGA, PCL poly(e-caprolactone), polyurethane, poly(ether ester).

3. Hydrogels natural materials, collagen, fibrin, proteoglycans (HA) chitosan, alginate, synthetic materials (PEG poly (ethylene glycol), PuraMatrix ${ }^{\mathrm{TM}}$ (peptide hydrogel), ${ }^{105,106}$ and self-assembling peptides (SAP).

4. Bioceramics (calcium phosphates, hydroxyapatite, tricalcium phosphate, biphasic calcium phosphate, bioactive silica-based glasses. ${ }^{104}$

Biomaterials utilized for dental pulp tissue engineering include PGA, type I and III collagens, alginate, chitosan, gelatin, and DMP1 in close association with collagen. Triple antibiotic paste, $\mathrm{Ca}(\mathrm{OH})_{2}$ and formocresol produced significantly greater increases in root length than either MTA apexification and non-surgical root canal treatment (NSRCT) used as a control groups. ${ }^{46}$ Smart scaffolds promote early capillary invasion via blood clotting, maintaining cell activity and phenotype, and inducing cell differentiation of existing progenitor cells. Self-assembling peptides (SAP) can be modified, and therefore address the various requirements for a specific tissue-engineering approach. They are usually synthesized at 15 to 25 amino acids in length, which undergo self-assembly, generate nanofibrous networks, entrap water, and may contribute to form hydrogels. Hydrogel systems offer advantages such as viscoelastic properties similar to those of soft connective tissues, fast diffusion of nutrients and metabolites, and the possibility of homogenous cell encapsulation. The use of multidomain peptides is furthermore advantageous because it relies on an automated synthesis process, high control over the material properties, a wide range of chemical functionalities, and the possibility for tailoring toward specific applications. Features such as matrix modulus, sheer recovery, cell adhesion motifs, enzymecleavable sites for cell-mediated degradation, controlled release of bioactive molecules, mineral nucleation, and antibacterial activity can be incorporated into the scaffold, and consequently making a matrix useful for pulp engineering.

The aims of the studies ${ }^{107}$ were to investigate the odontogenic differentiation of human dental pulp stem cells (DPSCs) on nanofibrous (NF) poly (L-lactic acid) (PLLA) scaffolds in vitro and in vivo. Highly porous NF-PLLA scaffolds which mimic the architecture of collagen type I fibers combined a phase separation technique and a porogen leaching method. They were fabricated by the combination of a phase separation technique and a porogen leaching method. Aligned PLGA/ Gelatin electrospun sheet (APES), treated dentin matrix (TDM) and native dental pulp extracellular matrix (DPEM) were constructed and combined into APES/TDM and pulp extracellular matrix (DPEM) TDM with the aim of dental pulp regeneration. ${ }^{108}$ The results suggest that APES and DPEM in conjunction with TDM, could make up an ECM microenvironment for tooth root regeneration, which also offers a strategy for complex tissue or organ regeneration. Native human teeth implanted in mouse dorsum deliver basic fibroblast growth factor and/or vascular endothelial growth factor (bFGF and/ or VEGF), yield recellularized and revascularized connective tissue that integrated native dentinal wall to the root canals. Combining delivery of bFGF, VEGF, or platelet-derived growth factor (PDGF) with nerve growth factor (NGF) and bone morphogenetic protein-7 (BMP7) cellularized and vascularized tissues were generated. They were positive of VEGF antibody staining, and neo-dentin formation over the surface of native dentinal wall. Newly formed dental pulp tissue appeared to be dense with disconnected cells surrounded by an extracellular matrix. ${ }^{109}$ To conclude, after alteration of the dental pulp due to endogenous (metallo)proteinases or resolving a dental pulp infection linked to exogenous carious bacteria, stem cells associated with growth factors and cell carriers may be implicated in root pulp repair and regeneration.

\section{Acknowledgments}

None.

\section{Conflicts of interest}

The author delcares that there is no conflicts of interest.

\section{References}

1. Baume IJ. The biology of pulp and dentin. In : Myers HM, editor. Monographs in Oral Sciences. 1980.

2. Fanibunda SB, A method of measuring the volume of human dental pulp cavities. Int Endod J. 1986;19:194-197.

3. Goldberg M. Pulp anatomy and characterization of pulp cells. In: Goldberg M, editor. The Dental Pulp Biology, pathology, and regenerative therapies. Springer-Verlag Berlin, Heidelberg. 2014a;13-33.

4. Goldberg M. Dentin, pulp and tooth pain. IN : Arthur Hand, Marion E Frank, editors. Fundamentals of oral histology and physiology. John Wiley Blackwell, Oxford University Press, New York, 2014b:85-112.

5. Philippas GG. Influence of occlusal wear and age on formation of dentin and size of pulp chamber. $J$ Dent Res. 1961;40:1186-1198.

6. Smith AJ, Cassidy N, Perry H. et al. Reactionary dentinogenesis Int $J$ Dev Biol.1995;39:273-280.

7. Bernick S. Age changes to the dental pulp. Oral morphological changes in older subjects. Front Oral Physiol. 1987;6:7-30.

8. Thomas HF. Root formation Int J Dev Biol.1995;39:231-237.

9. Takagi Y, Nagai H, Sasaki S. Difference in noncollagenous matrix composition between crown and root dentin of bovine incisor. Calcified Tissue Int. 1988;42(2):97-103.

10. Andujar MB, Couble P, Couble ML, et al. Differential expression of type I and type III collagen genes during tooth development. Development. 1991;111(3):691-698.

11. Goldberg M, Lasfargues JJ. Pulp-dentinal complex revisited. J Dent 1995;23(1):15-20.

12. Veis A, Goldberg M. Pulp extracellular matrix. In: Goldberg M, editor The dental pulp. Springer Verlag Berlin Heidelberg. 2014:35-46.

13. Love RM, Jenkinson HF. Invasion of dentinal tubules by oral bacteria. Crit Rev Oral Biol Med. 2002;13(2):171-183.

14. Schilke R, Lisson JA, Bauss O, et al. Comparison of the number and diameter of dentinal tubules in human and bovine dentine by scanning electron microscopic investigation. Arch Oral Biol. 2000;45(5):355-361.

15. Angelova A, Takagi Y, Okiji T, et al. Immunocompetent cells in the pulp of human deciduous teeth. Arch Oral Biol. 2004;49(1):29-36.

16. Zhang H, Jiang Y, Qin C, et al. Essential role of osterix for tooth rot but not crown dentin formation. J Bone Mineral Research. 2015;30:742-746. 
17. About I. Pulp vascularization and its regulation by the microenvironment In: Goldberg $\mathrm{M}$, editor. The dental pulp. Springer Verlag Berlin Heidelberg Chapter. 61-74.

18. Takahashi K. Vascular architecture of dog pulp using corrosion resin cast examined under a scanning electron microscope. J Dent Res. 1985;64:579-584.

19. Waddington RJ, Youde SJ, Lee CP. Sloan A.J. Isolation of distinct progenitor stem cell populations from DP. Cells Tissues Organs. 2009;189(1-4):268.

20. Kerkis I, Kerkis A, Dozortsev D, et al. Isolation and characterization of a population of immature dental pulp cells expressing OCT-4 and other embryonic stem cells markers. Cells Tissues Organs. 2006;184:105-116.

21. Närhi M, Jyväsjärvi E, Virtanan A, et al. Role of intradental A- and C-type nerve fibres in dental pain mechanisms. Proc Finn Dent Soc. 1992;88(Suppl 1):507-516.

22. Byers MR, Dong WK. Autoradiographic location of sensory nerve endings in dentin of monkey teeth. Anat Rec. 1983;205(4):441-454.

23. Byers MR. Dental sensory receptors. Int Rev Neurobiol. 1984;25:39-94.

24. Byers MR, Suzuki H, Maeda T. Dental neuroplasticity, neuro-pulpal interactions, and nerve regeneration Micr Res Tech. 2003;60(5):503-515.

25. Byers MR, Mecifi KB, Iadarola MJ. Int J Dev Biol. 1995;39:181-189.

26. Fearnhead RW. Histological evidence for the innervation of human dentine J Anat. 1957;91(2):267-277.

27. Brännström M. Physio-pathological aspects of dentinal and pulpal response to irritants. Dentine and pulp University of Dundee. 1968;231-246.

28. Heyraas KJ, Kvinnsland I, Byers MR, et al. Nerve fibers immunoreactive to protein gene product 9.5 , calcitonin gene-related peptide, substance $P$ and neuropeptide $\mathrm{Y}$ in the dental pulp, periodontal ligament, and gingiva in cats. Acta Odontologica Scandinavica. 1993;51(4):207-221.

29. Maeda T, Honma S, Takano Y. Dense innervation of human radicular dental pulp as revealed by immunocytochemistry for protein geneproduct 9.5. Arch oral Biol. 1994;39(7):563-568.

30. Arthur A, Rychkov, G, Shi S, et al. Adult human dental pulp stem cells differentiate toward functionally active neurons under appropriate environmental cues. Stem Cells. 2008;26(7):1787-1795.

31. Sonoyama W, Liu Y, Fang D, et al. Mesenchymal stem cell-mediated functional tooth regeneration in Swine. PLOS One. 2006;20(1):e79.

32. Sato O, Takeuchi-Maeno H, Maeda T, et al. Immunoelectron microscopic observation of calcitonin gene-related peptide (CGRP)-positive nerve in the dental pulp of rat molars Arch Histol Cytol. 1992;55:561-568.

33. Fried K. Changes in pulp nerves with aging. Proc Finn Dent Soc. 1992;88(Suppl):517-528.

34. Fried K, Gibbs JL. Dental pulp innervation. In: Goldberg, editor. The dental pulp. Springer Verlag Berlin Heidelberg. 2014;75-95.

35. Maeda T, Honma S, Takano Y. Dense innervation of human radicular dental pulp as revealed by immunocytochemistry for protein geneproduct 9.5 Arch Oral Biol. 1994;39(7):563-568.

36. Maeda $\mathrm{T}$, Iwanaga $\mathrm{T}$, Fujita $\mathrm{T}$, et al. Immunohistochemical demonstration of the nerves in human dental pulp with antisera agains neurofilament protein and gliaspecific S-100 protein. Arch Histol Jpn. 1985;48(1):123-129.

37. Rodd HD, Boissonade FM. Innervation of human tooth pulp in relation to caries and dentition type. J Dent Res. 2001;80(1):389-393.

38. Yu C, Abbott PV. An overview of the dental pulp : its functions and responses to injury. Australian Dent. J. Supplement. 2007;52(1) Suppl:S4-S16.
39. Lizier NF, Kerkis A, Gomes CM, et al. Scaling up of dental pulp stem cells isolated from multiple niches. PLOS One. 2012;7(6):e39885.

40. Marsh PD. Dental biofilm in health and disease. Springer Int Publishing Switzerland. 2016:41-52.

41. Tjäderhane L, Palossari H, Wahlgren J, et al. Human odontoblast culture method : the expression of collagen and matrix metalloproteinases (MMPs). Adv Dent Res. 2001;15:55-58.

42. Sulkala M, Larmas M, Sorsa $T$, et al. The localization of matrix metalloproteinase-20 (MMP-20, enamelysis) in mature human teeth. $J$ Dent Res. 2002;81(9):603-607.

43. Tersariol ILS, Geraldelt S, Minciotti CL, et al. Cysteine Cathepssins in human dentin-pulp complex. J Endod. 2010;36:475-481.

44. Nascimento FD, Minciotti CL, Geraldeli S, et al. Cysteine Cathepsins in human carious dentin. J Dent Res. 2011;90(4):506-511.

45. Goldberg M. Superficial and deep carious lesions. Chapter 9 In Understanding Dental Caries (M. Goldberg ed.) Springer Int Publishing Switzerland. 2016;85-96.

46. Bose R, Nummikoski P, Hargreaves K. A retrospective evaluation of radiographic outcomes in immature teeth with necrotic root canal systems treated with regenerative endodontic procedures. J Endod. 2009;35:1343-1349.

47. Huang GTJ, Yamaza T, Shea L, et al. Stem/progenitor cell-mediated de novo regeneration of dental pulp with newly deposited continuous layer of dentin in an in vivo model Tissue Engineering Part A. 2010;16(2):605-615.

48. Tatullo M, Marrelli M, Shakesheff KM, et al. Dental pulp stem cells: function, isolation and applications in regenerative medicine. $J$ Tissue Eng Regen Med. 2015;9(11):1205-1216.

49. Paino F, Ricci G, De Rosa A, et al. Ecto-mesenchymal stem cells from dental pulp are committed to differentiate into active melanocytes. Europ Cells and Materials. 2010;20:295-305.

50. Jontell M, Okiji T, Dahlgren U, et al. Immune defense menchanisms of the dental pulp. Crit Rev oral Biol Med. 1998;9(2):179-200.

51. MacDougall M, Simmons D, Luan X, et al. Dentin phosphoprotein and dentin sialoprotein are cleavage products expressed from a single transcript coded by a gene on human chromosome 4 . Dentin phosphoprotein DNA sequence determination. J Biol Chem. 1997;272(2):835-842.

52. Priam F, Ronco V, Locker M, et al. New cellular models for tracking the odontoblast phenotype. Arch Oral Biol. 2005;50(2):271-277.

53. Jontell M, Gunraj MN, Bergenholtz G. Immunocompeteent cells in the normal dental pulp. J Dent Res. 1987;66(6):1149-1153.

54. Jontell M, Bergenholtz G, Scheynius A, Ambrose W. Dendritic cells and macrophages expressing class II antigens in normal rat incisor pulp. $J$ Dent Res. 1988;67(10):1263-1266.

55. Zhao Z, Wen LY, Jin M, et al. ADAM28 participates in the regulation of tooth development. Arch Oral Biol. 2006;51(11):996-1005.

56. Zhao Z, Liu H, Wang D. ADAM28 manipulates proliferation, differentiation, and apoptosis of human dental pulp $J$ Endod. 2011;37(3):332-339.

57. Mitsiadis TA, De Bari C, About I. Apoptosis is developmental and repair-related human tooth remodeling : a view from the inside. Exp cell Research. 2008;314(4):869-877.

58. Nishikawa S, Sasaki F. Apoptosis of dental pulp cells and their elimination by macrophages and MHC ClassII-expressing dendritic cells. J Histochem Cytochem. 1999;47(3):303-311.

59. Satchell PG, Gutmann JL, Witherspoon DE. Apoptosis : an introduction for the endodontist. Int. Endodontic J. 2003;36(4):237-245. 
60. Wu TT, Li LF, Du R, et al. Hydrogen peroxide induces apoptosis in human dental pulp cells via Caspase-9 dependent pathway. $J$ Endod. 2013;39(9):1151-1155.

61. Mitsiadis TA, Feki A, Papaccio G, Caton J. Dental pulp stem cells, niches, and Notch signaling in tooth injury. Adv Dent Res. 2011;23(3):275-279.

62. Zhao Y, Wang L, Jin Y, et al. Fas Ligand regulate the immunomodulatory properties of dental pulp stem cells. J Dent Res. 2012;91(10):948-954.

63. Alvarez MMP, Moura GE, Machado MFM, et al. PAR-1 and PAR2 expression is enhanced in inflamed odontoblast cells. J Dent Res. 2017;96(13):1-8.

64. Wu Q, Qi S, Ma J, et al. The effect of NRAGE on cell cycle and apoptosis of human dental pulp cells and MDPC-23. Int J Clin Exp Med. 2015;8(7):10657-10667.

65. Saewong S, Thammasitboon K, Wattnaroonwong N. Simvastatin induces apoptosis and disruption of the actin cytoskeleton in human dental pulp cells and periodontal ligament fibroblasts. Arch Oral Biol. 2013;58(8):964-974.

66. Beertsen W, Niehof A. Root-analogue versus crown-analogue dentin : a radioautographic and ultrastructural investigation of the mouse incisor. Anat Rec. 1986;215(2):106-118.

67. Ogbureke KU, Fisher LW. Expression of SIBLINGs and their partner MMPs in salivary glands J Dent Res. 2004;83(9):654-670.

68. Ogbureke KUE, Fisher LW. Renal expression of SIBLING proteins and their partner matrix metalloproteinases (MMPs). Kidney Int. 2005;68(1):155-166.

69. Gronthos S, Brahim J, Li W, et al. Stem cell properties of human dental pulp stem cells. $J$ Dent Res. 2002;81(8):531-535.

70. Chaussain-Miller C, Fioretti F, Goldberg M, et al. The role of matrix metalloproteinases (MMPs) in human caries. J Dent Res. 2006;85(1):22-32.

71. Goldberg M, Septier D, Rapoport O, et al. Targeted disruption of two small leucine-rich proteoglycans, biglycan and decorin, excerpts divergent effects on enamel and dentin formation. Calcif. Tissue Int. 2005;77(5):297-310.

72. Goldberg M, Septier D, Oldberg A, et al. Fibromodulin-deficient mice display impaired collagen fibrillogenesis in predentin as well as altered dentin mineralization and enamel formation $J$ Histochem Cytochem. 2006;54(5):525-537.

73. Min KS, Kwon YY, Lee HJ, et al. Effects of proinflammatory cytokines on the expression of mineralization markers and heme Oxygenase-1 in human pulp cells. J Endod. 2006;32(1):39-43.

74. Shiba H, Nakamura S, Shirakawa M, N et al. Effects of basic Fibroblast Growth Factor on proliferation, the expression of osteonectin (SPARC) and alkaline phosphatase, and calcification in cultures of human pulp cells. Dev Biol. 1995;170:457-466.

75. Trantor IR, Messer HH, Bimer R. The effects of neuropeptiddes (calcitonin gene-related peptide and substance $\mathrm{P}$ ) on cultured human pulp cells. J Dent Res. 1995;74(4):1066-1071.

76. Imfeld T. Dental erosion. Definition, classification and links. Eur J Oral Sc.i 1996;104:151-155.

77. Selwitz RH, Ismail AI, Pitts NB. Dental caries. Lancet. 2007;369(9555):51-59.

78. Fejerskov O. Changing paradigms in concepts on dental caries : consequences for oral health care. Caries Res. 2004;38(3):182-191.

79. Featherstone JBD. Dental caries : a dynamic disease process. Australian Dental J. 2008;53:286-291.

80. Loesche WJ. Role of Streptococcus mutans in human dental decay. Microbiological Reviews. 1986;50(4):353-380.
81. Tanzer JM, Livingston J, Thompson AM. The microbiology of primary dental caries in human. JDental Education. 2001;65(10):(10):1028-1037.

82. Kidd EAM, Fejerskov O. What constitutes dental caries? Histopathology of carious enamel and dentin related to the action of cariogenic biofilms. J Dent Res. 2004;83(Spec Iss C):C35-C38.

83. Huang GTJ, Gronthos S, Shi S. Mesenchymal stem cells derived from dental tissues vs. those from other sources : their biology and role in regenerative medicine. J Dent Res. 2009;88(9):792-806.

84. Eminli S, Utikal J, Arnold K, et al. Reprogramming of neural progenitor cells into Induced Pluripotent Stem Cells in the absence of exogenous Sox2 expression. Stem cells. 2008;26(10):2467-2474.

85. Otsu K, Kumakami-Sakano M, Fujiwara K, et al. Stem cell sources for tooth regeneration: current status and future prospects. Front Physiol. 2014.

86. Takahashi K, Yamanaka S. Induction of pluripotent stem cells from mouse embryonic and adult fibroblast cultures by defined factors. Cell. 2006;126(4):663-676.

87. Tamaoki N, Takahashi K, Tanaka T, et al. Dental pulp cells for induced pluripotent stem cell banking. J Dent Res. 2010;89(8):773-778.

88. Okita K, Ichisaka T, Yamanaka S. Generation of germline-competent induced pluripotent stem cells. Nature. 2007;448(7151):313-317.

89. Oda Y, Yoshimura Y, Ohnishi H, et al. Induction of pluripotent stem cells from human third molar mesenchymal stromal cells. Journal Biological Chemistry. 2010;285:29270-29278.

90. Kang L, Kou Z, Zhang Y, et al. Induced pluripotent stem cells (iPSCs)a new area of reprogramming $J$ Genet Genomics. 2010;37(7):415-421.

91. Potdar P, Jethmalani YD. Human dental pulp stem cells: applications in future regenerative medicine. World J Stem Cells. 2015;7(5):839-851.

92. Silva J, Nichols J, Theunissen TW, et al. Nanog is the gateway to the pluripotent ground state. Cell. 2009;138(4):722-737.

93. Iohara K, Zheng L,Wake Ito M, et al. Side population cells isolated from porcine dental pulp tissue with self $\square$ renewal and multipotency for dentinogenesis, chondrogenesis, adipogenesis , and neurogenesis Stem cells. 2006;24(11):2493-2503.

94. Huang GTJ. Pulp and tissue engineering and regeneration: current progress. Regen Med. 2009;4(5):697-707.

95. Nakashima M, Iohara K. Mobilized dental pulp stem cells for pulp regeneration: initiation of clinical trial. J Endod. 2014;40 (4 Suppl):526-532.

96. Iohara K, Nakashima M, Ito M, et al. Dentin regeneration by dental pulp stem cell therapy with recombinant human bone Morphogenetic Protein 2. J Dent Res. 2004;83(8):590-595.

97. Iohara $\mathrm{K}$, Zheng $\mathrm{L}$, Wake $\mathrm{H}$, et al. A novel stem cell source for vasculogenesis in ischemia: subfraction of side population cells from dental pulp. Stem cells. 2008;26(9):2408-2418.

98. Iohara K, Imabayashi K, Ishizaka R, et al. Complete pulp regeneration after pulpectomy by transplantation of CD105+ stem cells with stromal cell-derived factor-1. Tissue Engineering Part A. 2011;17(1516):1911-1930.

99. Colombo JS, Moore AN, Hartgerink JD, et al. Scaffolds to control inflammation and facilitate dental pulp regeneration. J Endod. 2014;40(4 Suppl):S6-S12.

100. Serhan CN, Clish CB, Brannon J, et al. Novel functional sets of lipid-derived mediators with anti-inflammatory actions generated from Omega-3 Fatty Acids via Cyclooxygenase 2-nonsteroidal anti-inflammatory drugs and transcellular processing. J Exp Med. 2000;192(8):1197-1204.

101. Serhan CN, Petasis NA. Resolvins and protectins in inflammation resolution. Chem Rev. 2011;11(10):5922-5943. 
102. Xu ZZ, Zhang L, Lui T, et al. Resolvins RvE1 and RvD1 attenuate inflammatory pain via central and peripheral cations. Nat $\mathrm{Med}$. 2010;16(5):592-597.

103. Galler K, Eidt A, Schmalz G. Cell-free approaches for dentaal pulp engineering. J Endodod. 2014;40(4 Suppl):541-545.

104. Galler KM, D’Souza RN, Hartgerink JD, et al. Scaffolds for dental pulp tissue engineering. Adv Dent Res. 2011;23(3):333-339.

105. Rosa V, Zhang Z, Grande RHM, Nör JE. Dental pulp tissue engineering in full-length human root canals. J Dent Res 2013:92(11):970-975.

106. Dissanayaka WL, Hargreaves KM, Jin L, et al. The interplay of dental pulp stem cells in an injectable peptide hydrogel on angiogenesis and pulp regeneration in vivo. Tissue Engineering Part A. 2015;21(3 \& 4):550-563.
107. Wang J, Liu X, Jin X, et al. The odontogenic differentiation of human dental pulp stem cells on nanofibrous poly(L-lactic acid) scaffolds in vitro and in vivo. Acta Biomater. 2010;6(10):3856-3863.

108. Chen G, Chen J, Yang B, et al. Combination of aligned PLGA/Gelatin electrospun sheets, native dental pulp extracellular matrix and treated dentin matrix as substrates for tooth root regeneration. Biomaterials. $2015 ; 52: 56-70$.

109. Kim JY, Xin X, Moioli EK, et al. Regeneration of dental-pulp-like tissue by chemotaxis-induced cell homing. Tiss Engenieering Part A. 2010;16(10):3023-3031. 